\title{
An Approach to the Geometric-Arithmetic Index for Graphs under Transformations' Fact over Pendent Paths
}

\author{
Muhammad Asif $\left(\mathbb{D},{ }^{1}\right.$ Hamad Almohamedh $\left(\mathbb{D},{ }^{2}\right.$ Muhammad Hussain $(\mathbb{D}){ }^{1}$ \\ Khalid M Alhamed $\mathbb{D},{ }^{3}$ Abdulrazaq A. Almutairi $\mathbb{D}^{4},{ }^{4}$ and Sultan Almotairi $\mathbb{D D}^{5}$ \\ ${ }^{1}$ Department of Mathematics, COMSATS University Islamabad, Lahore Campus, Lahore 54000, Pakistan \\ ${ }^{2}$ King Abdulaziz City for Science and Technology (KACST) Riyadh, Riyadh, Saudi Arabia \\ ${ }^{3}$ IT Programs Center, Faculty of IT Department, Institute of Public Administration, Riyadh 11141, Saudi Arabia \\ ${ }^{4}$ Information and Computer Center, The Public Authority for Applied Education and Training, The Ministry of Education, \\ Kuwait, Kuwait \\ ${ }^{5}$ Department of Natural and Applied Sciences, Faculty of Community College, Majmaah University, \\ Majmaah 11952, Saudi Arabia
}

Correspondence should be addressed to Hamad Almohamedh; halmohamedh@kacst.edu.sa and Sultan Almotairi; almotairi@mu.edu.sa

Received 10 May 2021; Accepted 12 June 2021; Published 24 June 2021

Academic Editor: Muhammad Javaid

Copyright (C) 2021 Muhammad Asif et al. This is an open access article distributed under the Creative Commons Attribution License, which permits unrestricted use, distribution, and reproduction in any medium, provided the original work is properly cited.

\begin{abstract}
Graph theory is a dynamic tool for designing and modeling of an interconnection system by a graph. The vertices of such graph are processor nodes and edges are the connections between these processors nodes. The topology of a system decides its best use. Geometric-arithmetic index is one of the most studied graph invariant to characterize the topological aspects of underlying interconnection networks or graphs. Transformation over graph is also an important tool to define new network of their own choice in computer science. In this work, we discuss transformed family of graphs. Let $\Gamma_{n}^{k, l}$ be the connected graph comprises by $k$ number of pendent path attached with fully connected vertices of the $n$-vertex connected graph $\Gamma$. Let $A_{\alpha}\left(\Gamma_{n}^{k, l}\right)$ and $A_{\alpha}^{\beta}\left(\Gamma_{n}^{k, l}\right)$ be the transformed graphs under the fact of transformations $A_{\alpha}$ and $A_{\alpha}^{\beta}, 0 \leq \alpha \leq l, 0 \leq \beta \leq k-1$, respectively. In this work, we obtained new inequalities for the graph family $\Gamma_{n}^{k, l}$ and transformed graphs $A_{\alpha}\left(\Gamma_{n}^{k, l}\right)$ and $A_{\alpha}^{\beta}\left(\Gamma_{n}^{k, l}\right)$ which involve GA $(\Gamma)$. The presence of $\mathrm{GA}(\Gamma)$ makes the inequalities more general than all those which were previously defined for the GA index. Furthermore, we characterize extremal graphs which make the inequalities tight.
\end{abstract}

\section{Introduction}

The advancement in technology mainly networking, computer, biological, and electrical networks made practicable the accurate data transfer within very small duration. The Internet, social media, biological, ecological, and neural networks are few examples of such networks. Telecommunication is based on interconnection networks which used to share data files. Similarly, data exchange using computing devices is also based on computer network through data linkage, optical fiber cable (OFC), and wireless media such as Wi-Fi. Different algorithms are used for directing, arranging/determining numerical calculations, and image processing. Multiprocessor interconnection networks (MINs) are used to design powerful microprocessors and memory chips $[1,2]$.

Graph theory provides a fundamental tool for designing and analyzing such networks. Naturally, the interconnection system is modeled by the graph with processor nodes as vertices and links between these nodes as edges of such graph. Graph theory and interconnection networks provide a thorough understanding of these interrelated topics through their topology. The topology of a graph provides information about the manner in which vertices joined in a graph. The topological indices are graph invariants used to study the topology of graphs. Other than computer 
networks, graph theory is considered as a powerful tool in different areas of research, such as in coding theory, database management system, circuit design, secret sharing schemes, and theoretical chemistry [3]. The topological descriptors of several interconnection networks are already been computed in [4-6]. Along with interconnection networks, these invariants are equally important in chemical graph theory which deals with problems in chemistry using associated graph of chemical compounds [7].

The study of underlying substance using their graph with the help of graph invariants plays an important role in cheminformatics, pharmaceutical sciences, materials science, engineering, and so forth [8,9]. Among theoretical molecular descriptors, topological indices have an impact in chemistry due to the prediction of physio-chemical properties of the underlying substance. Its role in the QSPR/ QSAR analysis to model physical and chemical properties of molecules is also remarkable [10-12]. Actually, topological indices are designed on the ground of transformation which associates a numeric value with the graph which characterizes its topology [13]. The first topological index, named Winner index, was proposed in 1947 by Winner [14]. It provides best correlation with the boiling points of alkanes. The discovery of the Winner index provides emerging research platform to the research community. The interest in accurate prediction of physio-chemical properties encouraged the researchers to propose a large class of topological indices. For the first time, an index is defined on the base of end vertices' degrees of the edges by Milan Randić named Randić connectivity index [15]:

$$
\operatorname{RA}(\Gamma)=\sum_{u v \in E(\Gamma)} \frac{1}{\sqrt{\operatorname{deg}_{u} \operatorname{deg}_{v}}} .
$$

Due to this reason, it has attained a great attraction of the researchers till now. In 2009, Vukičević and Furtula [16] introduced the geometric-arithmetic index:

$$
\mathrm{GA}(\Gamma)=\sum_{s t \in E(G)} \frac{2 \sqrt{\operatorname{deg}_{s} \operatorname{deg}_{t}}}{\operatorname{deg}_{s}+\operatorname{deg}_{t}}
$$

GA has correlation coefficient of 0.972 with heat of formation of benzene hydrocarbons. Also, in case of "standard enthalpy of vaporization," its accuracy is $9 \%$ more than the Randić index. Due to this reason, GA was studied more than all other indices in the last decade. The bonds and extremal characterization of graphs regarding the GA index were studied at some extent in [17-24]. It encouraged us to study the GA index for $\Gamma_{n}^{k, l}$ and transformed graphs $A_{\alpha}\left(\Gamma_{n}^{k, l}\right)$ and $A_{\alpha}^{\beta}\left(\Gamma_{n}^{k, l}\right)$ under the fact of transformations $A_{\alpha}$ and $A_{\alpha}^{\beta}$, $0 \leq \alpha \leq l 0 \leq \beta \leq k-1$, respectively. We characterize extremal graphs for all of these families of graphs.

\section{Results and Discussion}

Throughout this work, let graph $\Gamma_{n}^{k, l}$ comprise with $n$-vertex simple connected graph $\Gamma$ along with $k$ pendent paths of length $l \geq 2$ attached with $v \in \Gamma$, having degree $d_{v} \geq 2$. The order of $\Gamma_{n}^{k, l}$ is $n+k l$, size is $m+k l$, and $\operatorname{deg}_{1}=\delta_{\Gamma} \leq \operatorname{deg}_{2} \leq \operatorname{deg}_{3} \leq \cdots \leq \Delta_{\Gamma}+1$ is its degree sequence.

Let graph $\Gamma=\Gamma(V, E)$ be with the degree of vertex $u \in \Gamma$ and $\delta_{\Gamma} \leq \operatorname{deg}_{u} \leq \Delta_{\Gamma}$ and $\delta_{\Gamma} \leq \operatorname{deg}_{v} \leq \Delta_{\Gamma}+1$ be the degrees of $v \in \Gamma_{n}^{k, l}$. For validity of our proved results, we defined the following list of useful graphs.

Type I: let $\delta_{\Gamma} \leq \operatorname{deg}_{u} \leq \Delta_{\Gamma}$, where $u \in V(\Gamma) . \Gamma_{n}^{k, l}$ of type I is obtained by attaching pendent paths of length $l$ with vertices of degree $\operatorname{deg}_{u} \geq 2$ in such a way that the vertices with pendent path are adjacent to the vertices without pendent paths.

The graph of type I is shown in Figure 1(a).

Type II: $\Gamma_{n}^{k, l}$ of type II is the graph of type I with $\operatorname{deg}_{u}=\Delta_{\Gamma}, \forall u \in V(\Gamma)$.

The graph of type II is shown in Figure 1(b).

Before attempting the major problem, we prove the following preposition.

Proposition 1. Let $x \geq 2$; then,

$$
\frac{2 \sqrt{x(x-1)}}{2 x-1} \leq \frac{2 \sqrt{x(x+1)}}{2 x+1} \text {. }
$$

Proof. Let $x \geq 2$ :

$$
\begin{aligned}
& \frac{2 \sqrt{x(x+1)}}{2 x+1}-\frac{2 \sqrt{x(x-1)}}{2 x-1} \\
& =\frac{2 \sqrt{x(x+1)}(2 x-1)-2 \sqrt{x(x-1)}(2 x+1)}{(2 x+1)(2 x-1)} \\
& =\frac{2 \sqrt{x}[x \sqrt{(x+1)}+(x-1) \sqrt{(x+1)}-x \sqrt{(x-1)}-(x+1) \sqrt{(x-1)}]}{(2 x+1)(2 x-1)} \\
& =\frac{2 \sqrt{x}\left[\sqrt{x+1}\left(x-\sqrt{x^{2}-1}\right)-\sqrt{x-1}\left(x-\sqrt{x^{2}-1}\right)\right]}{(2 x+1)(2 x-1)} \\
& =\frac{2 \sqrt{x}\left(x-\sqrt{x^{2}-1}\right)(\sqrt{x+1}-\sqrt{x-1})}{(2 x+1)(2 x-1)} \geq 0 .
\end{aligned}
$$




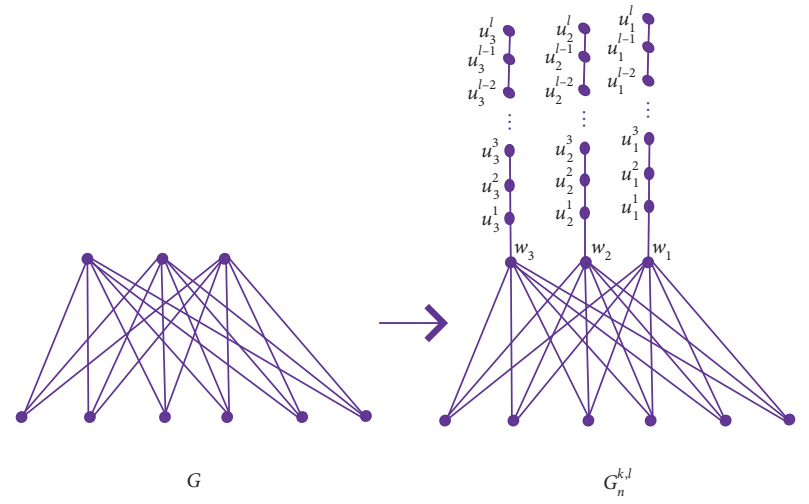

(a)

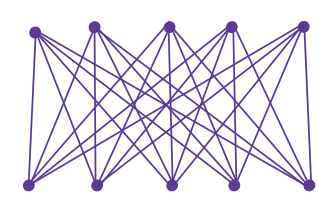

G

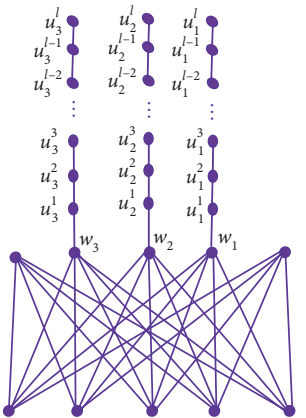

$G_{n}^{k, l}$

(b)

Figure 1: Graph $\Gamma_{n}^{k, l}$. (a) Graph $\Gamma_{n}^{k, l}$ of type I. (b) Graph $\Gamma_{n}^{k, l}$ of type II.

The above calculations implies

$$
\frac{2 \sqrt{x(x-1)}}{2 x-1} \leq \frac{2 \sqrt{x(x+1)}}{2 x+1} \text {. }
$$

Theorem 1. Let graph $\Gamma_{n}^{k, l}$ comprise of n-vertex simple connected graph $\Gamma$ along with $k$ pendent paths of length $l \geq 2$ attached with $v \in \Gamma$ of degree $d_{v} \geq 2$, maximum degree $\Delta_{\Gamma}+1$, and minimum $\delta_{\Gamma}$. Then,

$$
\mathrm{GA}\left(\Gamma_{n}^{k, l}\right) \geq k\left[\frac{3\left(l-\Delta_{\Gamma}-2\right)+2 \sqrt{2}}{3}+2 \sqrt{\Delta_{\Gamma}+1}\left(\frac{\sqrt{2}}{\left(\Delta_{\Gamma}+3\right)}+\frac{\Delta_{\Gamma} \sqrt{\delta_{\Gamma}}}{\left(\Delta_{\Gamma}+\delta_{\Gamma}+1\right)}\right)\right]+\mathrm{GA}(\Gamma)
$$

Equality holds for graphs of type II. And,

$$
\mathrm{GA}\left(\Gamma_{n}^{k, l}\right) \leq k\left[\frac{2 \sqrt{2}+3 l-6}{3}+\frac{2 \sqrt{2\left(\delta_{\Gamma}+1\right)}}{\delta_{\Gamma}+3}+2 k \Delta_{\Gamma}^{3 / 2}\left(\frac{\sqrt{\Delta_{\Gamma}+1}}{2 \Delta_{\Gamma}+1}-\frac{\sqrt{\delta_{\Gamma}}}{\delta_{\Gamma}+\Delta_{\Gamma}}\right)\right]+\mathrm{GA}(\Gamma) .
$$

Equality holds for graph of type II.

Proof. Let a simple graph $\Gamma$ be of order $n$, size $m$, maximum degree $\Delta_{\Gamma}$, and minimum $\delta_{\Gamma}$. $\Gamma_{n}^{k, l}$ be the graph formed by $k$ number of paths having length $l$ pendent at distinct vertices $u \in \Gamma$ such that $2 \leq \operatorname{deg}_{u} \leq \delta_{\Gamma}$. The geometric-arithmetic index of any graph $\Gamma$ is

$$
\mathrm{GA}(\Gamma)=\sum_{s t \in E(\Gamma)} \frac{2 \sqrt{\operatorname{deg}_{s} \operatorname{deg}_{t}}}{\operatorname{deg}_{s}+\operatorname{deg}_{t}} .
$$

The construction of $\Gamma_{n}^{k, l}, l \geq 2$ implies $\left|E\left(\Gamma_{n}^{k, l}\right)\right|=m+k l$ and for $s t \in E\left(\Gamma_{n}^{k, l}\right)\left(\operatorname{deg}_{s}+\operatorname{deg}_{t}\right) \in\left\{3,4, \operatorname{deg}_{u}+2, \operatorname{deg}_{u}+\operatorname{deg}_{v}\right.$, $\left.\operatorname{deg}_{u}+\operatorname{deg}_{v}+1\right\}$.
The edge set of $\Gamma_{n}^{k, l}$ partitioned as

$$
\begin{aligned}
A_{3} & =\left\{s t \in \Gamma_{n}^{k, l}: \operatorname{deg}_{s}=1, \operatorname{deg}_{t}=2\right\}, \\
A_{4} & =\left\{s t \in \Gamma_{n}^{k, l}: \operatorname{deg}_{s}=\operatorname{deg}_{t}=2\right\}, \\
A_{\operatorname{deg}_{u}+2} & =\left\{s t \in \Gamma_{n}^{k, l}: \delta_{\Gamma} \leq \operatorname{deg}_{s}=\operatorname{deg}_{u} \leq \Delta_{\Gamma}, \operatorname{deg}_{t}=2\right\}, \\
A_{\operatorname{deg}_{u}+\operatorname{deg}_{v}} & =\left\{s t \in \Gamma_{n}^{k, l}: \delta_{\Gamma} \leq \operatorname{deg}_{s}=\operatorname{deg}_{u}, \operatorname{deg}_{t}=\operatorname{deg}_{v} \leq \Delta_{\Gamma}\right\},
\end{aligned}
$$

and $\quad A_{\operatorname{deg}_{u}+\operatorname{deg}_{v}+1}=\left\{s t \in \Gamma_{n}^{k, l}: \delta_{\Gamma} \leq \operatorname{deg}_{s}=\operatorname{deg}_{u}, \operatorname{deg}_{t}=\operatorname{deg}_{v}+1\right.$ $\left.\leq \Delta_{\Gamma}\right\}$, 


$$
\begin{aligned}
\mathrm{GA}\left(\Gamma_{n}^{k, l}\right)= & \sum_{\substack{\text { st are edges of } \\
\text { pendent paths }}} \frac{2 \sqrt{\operatorname{deg}_{s} \operatorname{deg}_{t}}}{\operatorname{deg}_{s}+\operatorname{deg}_{t}}+\sum_{\text {st are edges of } \Gamma} \frac{2 \sqrt{\operatorname{deg}_{s} \operatorname{deg}_{t}}}{\operatorname{deg}_{s}+\operatorname{deg}_{t}}, \\
\mathrm{GA}\left(\Gamma_{n}^{k, l}\right)= & \sum_{s t \in A_{3}} \frac{2 \sqrt{\operatorname{deg}_{s} \operatorname{deg}_{t}}}{\operatorname{deg}_{s}+\operatorname{deg}_{t}}+\sum_{s t \in A_{4}} \frac{2 \sqrt{\operatorname{deg}_{s} \operatorname{deg}_{t}}}{\operatorname{deg}_{s}+\operatorname{deg}_{t}}+\sum_{s t \in A_{\operatorname{deg}_{u}+3}} \frac{2 \sqrt{\operatorname{deg}_{s} \operatorname{deg}_{t}}}{\operatorname{deg}_{s}+\operatorname{deg}_{t}} \\
& +\sum_{s t \in A_{\operatorname{deg}_{u}+\operatorname{deg}_{v}+1}} \frac{2 \sqrt{\operatorname{deg}_{s} \operatorname{deg}_{t}}}{\operatorname{deg}_{s}+\operatorname{deg}_{t}}+\sum_{s t \in A_{\operatorname{deg}_{u}+\operatorname{leg}_{v}}} \frac{2 \sqrt{\operatorname{deg}_{s} \operatorname{deg}_{t}}}{\operatorname{deg}_{s}+\operatorname{deg}_{t}}
\end{aligned}
$$

The construction of GA $\left(\Gamma_{n}^{k, l}\right)$ implies that the cardinality of $A_{3} \quad$ is $k$, i.e., $\left|A_{3}\right|=k,\left|A_{4}\right|=k(l-2), \quad\left|A_{\operatorname{deg}_{u}+3}\right|=k$, $\left|A_{\operatorname{deg}_{u}+\operatorname{deg}_{u}+1}\right| \leq k \Delta_{\Gamma}$, and $\left|A_{\operatorname{deg}_{u}+\operatorname{deg}_{u}}\right| \leq k \Delta_{\Gamma}$. The function $f(x)$

$=2 \sqrt{a x} / a+x$ is decreasing, where $a \leq x$ is a constant. So, for $\delta_{\Gamma}$ minimum degree of vertices of $\Gamma$ and maximum degree $\Delta_{\Gamma}$, we have

$$
\frac{2 \sqrt{2 *\left(\operatorname{deg}_{s}+1\right)}}{2+\left(\operatorname{deg}_{s}+1\right)} \geq \frac{2 \sqrt{2 *\left(\Delta_{\Gamma}+1\right)}}{2+\left(\Delta_{\Gamma}+1\right)}, \frac{2 \sqrt{\left(\Delta_{\Gamma}+1\right) \delta_{\Gamma}}}{\left(\Delta_{\Gamma}+1\right)+\delta_{\Gamma}} \leq \frac{2 \sqrt{\left(\operatorname{deg}_{s}+1\right) \operatorname{deg}_{t}}}{\left(\operatorname{deg}_{s}+1\right)+\operatorname{deg}_{t}}, \frac{2 \sqrt{\operatorname{deg}_{s}+\operatorname{deg}_{t}}}{\operatorname{deg}_{s}+\operatorname{deg}_{t}} \geq \operatorname{GA}(\Gamma)-\Delta_{\Gamma} k
$$

From equation (10), we have

$$
\mathrm{GA}\left(\Gamma_{n}^{k, l}\right) \geq \frac{2 k \sqrt{2}}{3}+k(l-2)+\frac{2 k \sqrt{2 *\left(\Delta_{\Gamma}+1\right)}}{2+\left(\Delta_{\Gamma}+1\right)}+\frac{2 \Delta_{\Gamma} k \sqrt{\delta_{\Gamma}\left(\Delta_{\Gamma}+1\right)}}{\delta_{\Gamma}+\Delta_{\Gamma}+1}-k \Delta_{\Gamma}+\mathrm{GA}(\Gamma)
$$

After simplification, we obtain

$$
\mathrm{GA}\left(\Gamma_{n}^{k, l}\right) \geq k\left[\frac{3\left(l-\Delta_{\Gamma}-2\right)+2 \sqrt{2}}{3}+2 \sqrt{\Delta_{\Gamma}+1}\left(\frac{\sqrt{2}}{\left(\Delta_{\Gamma}+3\right)}+\frac{\Delta_{\Gamma} \sqrt{\delta_{\Gamma}}}{\left(\Delta_{\Gamma}+\delta_{\Gamma}+1\right)}\right)\right]+\mathrm{GA}(\Gamma)
$$

Now, again set

$$
\begin{aligned}
\frac{2 \sqrt{2 *\left(\operatorname{deg}_{s}+1\right)}}{2+\left(\operatorname{deg}_{s}+1\right)} & \leq \frac{2 \sqrt{2 *\left(\delta_{\Gamma}+1\right)}}{2+\left(\delta_{\Gamma}+1\right)}, \sum_{s t \in A_{\operatorname{deg}_{u}+\operatorname{deg}_{v}+1}} \frac{2 \sqrt{\operatorname{deg}_{s} \operatorname{deg}_{t}}}{\operatorname{leg}_{s}+\operatorname{deg}_{t}}+\sum_{s t \in A_{\operatorname{deg}_{u}+\operatorname{deg}_{v}}} \frac{2 \sqrt{\operatorname{deg}_{s} \operatorname{deg}_{t}}}{\operatorname{deg}_{s}+\operatorname{deg}_{t}} \\
& \leq \frac{2 \sqrt{\left(\Delta_{\Gamma}+1\right) \Delta_{\Gamma}}}{\left(\Delta_{\Gamma}+1\right)+\Delta_{\Gamma}}+\mathrm{GA}(\Gamma)-\frac{2 k \Delta_{\Gamma} \sqrt{\delta_{\Gamma} \Delta_{\Gamma}}}{\delta_{\Gamma}+\Delta_{\Gamma}},
\end{aligned}
$$

which implies from Proposition 1 and the characteristics of $f(x)=2 \sqrt{x y} / x+y$ in equation (10). We get the following inequality:

$$
\mathrm{GA}\left(\Gamma_{n}^{k, l}\right) \leq \frac{2 k \sqrt{2}}{3}+k(l-2)+\frac{2 k \sqrt{2 *\left(\delta_{\Gamma}+1\right)}}{2+\left(\delta_{\Gamma}+1\right)}+\frac{2 \Delta_{\Gamma} k \sqrt{\Delta_{\Gamma}\left(\Delta_{\Gamma}+1\right)}}{\Delta_{\Gamma}+\Delta_{\Gamma}+1}+\mathrm{GA}(\Gamma)-\frac{2 \Delta_{\Gamma} k \sqrt{\delta_{\Gamma} \Delta_{\Gamma}}}{\delta_{\Gamma}+\Delta_{\Gamma}} .
$$


After simplification, we obtain

$$
\mathrm{GA}\left(\Gamma_{n}^{k, l}\right) \leq k\left[\frac{2 \sqrt{2}+3 l-6}{3}+\frac{2 \sqrt{2\left(\delta_{\Gamma}+1\right)}}{\delta_{\Gamma}+3}+2 k \Delta_{\Gamma}^{3 / 2}\left(\frac{\sqrt{\Delta_{\Gamma}+1}}{2 \Delta_{\Gamma}+1}-\frac{\sqrt{\delta_{\Gamma}}}{\delta_{\Gamma}+\Delta_{\Gamma}}\right)\right]+\mathrm{GA}(\Gamma)
$$

Inequalities (13) and (16) complete the proof.

Corollary 1 shows generalization of the above defined inequalities. One can get more inequalities of their desire by replacing $\mathrm{GA}(\Gamma)$ with already defined bonds of the GA index.
Corollary 1. Let graph $\Gamma_{n}^{k, l}$ comprise of $n$-vertex simple connected graph $\Gamma$ along with $k$ pendent paths of length $l \geq 2$ attached with $v \in \Gamma$ of degree $d_{v} \geq 2$, maximum degree $\Delta_{\Gamma}+1$, and minimum $\delta_{\Gamma}$. Then,

$$
\begin{aligned}
& \mathrm{GA}\left(\Gamma_{n}^{k, l}\right) \geq k\left[\frac{3\left(l-\Delta_{\Gamma}-2\right)+2 \sqrt{2}}{3}+2 \sqrt{\Delta_{\Gamma}+1}\left(\frac{\sqrt{2}}{\left(\Delta_{\Gamma}+3\right)}+\frac{\Delta_{\Gamma} \sqrt{\delta_{\Gamma}}}{\left(\Delta_{\Gamma}+\delta_{\Gamma}+1\right)}\right)\right]+\frac{2 m \sqrt{\Delta_{\Gamma} \delta_{\Gamma}}}{\delta_{\Gamma}+\Delta_{\Gamma}} \\
& \mathrm{GA}\left(\Gamma_{n}^{k, l}\right) \leq k\left[\frac{2 \sqrt{2}+3 l-6}{3}+\frac{2 \sqrt{2\left(\delta_{\Gamma}+1\right)}}{\delta_{\Gamma}+3}+2 k \Delta_{\Gamma}^{3 / 2}\left(\frac{\sqrt{\Delta_{\Gamma}+1}}{2 \Delta_{\Gamma}+1}-\frac{\sqrt{\delta_{\Gamma}}}{\delta_{\Gamma}+\Delta_{\Gamma}}\right)\right]+m .
\end{aligned}
$$

Equality holds for regular graph of the type II.

Proof. Using results of Theorem 1 and inequality regarding the geometric index proved in $[25,26]$, as

$$
\frac{2 m \sqrt{\Delta_{\Gamma} \delta_{\Gamma}}}{\Delta_{\Gamma}+\delta_{\Gamma}} \leq \mathrm{GA}(\Gamma) \leq m,
$$

we get desired results.

2.1. Graph Transformations. Let $H(\Gamma) \subset E(\Gamma), \Gamma^{\prime}=\Gamma-H$ be the new graph generated by removing set edges of $H(\Gamma)$, and $\Gamma^{\prime \prime}=\Gamma-V_{1}(\Gamma)$ be the new graph generated by deleting set of vertices $V_{1}(\Gamma) \subset V(\Gamma)$. We use the following transformations as used in [27]. These transformations have solid effect over GA of $\Gamma_{n}^{k, l}$.
Transformation $\quad A$ : let $\quad w_{j} \in V(\Gamma), \operatorname{deg}_{w_{1}} \geq 2, \quad$ for $1 \leq j \leq k \leq n$, and paths pendent at $w_{j}$ of the form $\left\{w_{j} u_{j}^{1}, u_{j}^{1} u_{j}^{2}, u_{j}^{2} u_{j}^{3}, \ldots, u_{j}^{l-1} u_{j}^{l}\right\}$ comprise $\Gamma_{n}^{k, l}$. Then,

$$
\begin{aligned}
A\left(\Gamma_{n}^{k, l}\right)= & \Gamma_{n}^{k, l}-\sum_{j=1}^{k}\left\{u_{j}^{2} u_{j}^{3}, u_{j}^{3} u_{j}^{4}, \ldots, u_{j}^{l-1} u_{j}^{l}\right\} \\
& +\sum_{j=1}^{k}\left\{w_{j} u_{j}^{2}, u_{j}^{2} u_{j}^{3}, \ldots, u_{j}^{l-1} u_{j}^{l}\right\} .
\end{aligned}
$$

The transformation $A$ is shown in Figure 2.

In Theorem 2, we discuss the effect of transformation $A$ over the GA index.

Theorem 2. Let graph $\Gamma_{n}^{k, l}$ comprise of $n$-vertex simple connected graph $\Gamma$ along with $k$ pendent paths of length $l \geq 2$ attached with $v \in \Gamma$ of degree $d_{v} \geq 2$, maximum degree of $v \in \Gamma_{n}^{k, l}$ is $\Delta_{\Gamma}+1$, and minimum $\delta_{\Gamma}$. Then,

$$
\begin{aligned}
\mathrm{GA}\left(A_{\alpha}\left(\Gamma_{n}^{k, l}\right)\right) \geq & 2 k \sqrt{\Delta_{\Gamma}+\alpha+1}\left[\frac{\alpha}{\Delta_{\Gamma}+\alpha+2}+\frac{\sqrt{2}}{\Delta_{\Gamma}+\alpha+3}+\frac{\Delta_{\Gamma} \sqrt{\delta_{\Gamma}}}{\left(\Delta_{\Gamma}+1+\alpha\right)+\delta_{\Gamma}}\right] \\
+ & \frac{2 k \sqrt{2}}{3}+\mathrm{GA}(\Gamma)+k l-k\left(2+\alpha+\Delta_{\Gamma}\right) .
\end{aligned}
$$

Equality holds for all graphs of type II:

$$
\begin{aligned}
\mathrm{GA}\left(A_{\alpha}\left(\Gamma_{n}^{k, l}\right)\right) \leq & 2 k \sqrt{\delta_{\Gamma}+\alpha+1}\left[\frac{\alpha}{\delta_{\Gamma}+\alpha+2}+\frac{\sqrt{2}}{\delta_{\Gamma}+\alpha+3}\right]+\frac{2 k \sqrt{2}}{3} \\
& +k(l-2-\alpha)+\mathrm{GA}(\Gamma)+2 k \Delta_{\Gamma}^{3 / 3}\left[\frac{\sqrt{\Delta_{\Gamma}+1}}{2 \Delta_{\Gamma}+1}-\frac{\sqrt{\delta_{\Gamma}}}{\Delta_{\Gamma}+\delta_{\Gamma}}\right] .
\end{aligned}
$$




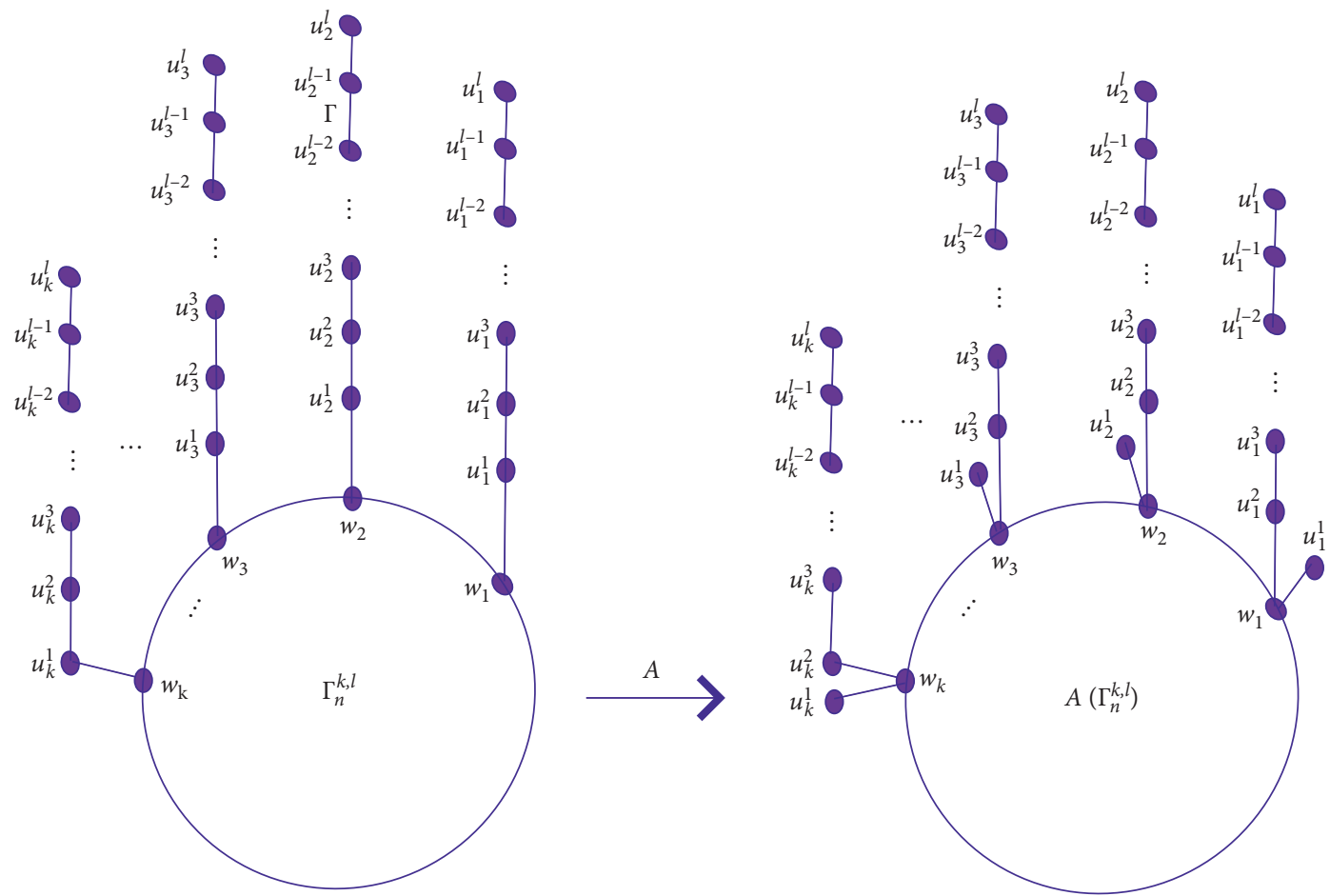

FIgURE 2: Transformation $A$.

Equality holds for all graphs of the type II and $\alpha=0$.

Proof. Let a simple graph $\Gamma$ be of order $n$, size $m$, minimum degree $\delta_{\Gamma}$, and maximum $\Delta_{\Gamma}$. Let $\Gamma_{n}^{k, l}$ be the graph formed by $k$ number of paths of length $l$ pendent at distinct fully connected vertices of $\Gamma$. The geometric-arithmetic index of any graph $\Gamma$ is

$$
\mathrm{GA}(\Gamma)=\sum_{s t \in E(G)} \frac{2 \sqrt{\operatorname{deg}_{s} \operatorname{deg}_{t}}}{\operatorname{deg}_{s}+\operatorname{deg}_{t}}
$$

The construction of $\Gamma_{n}^{k, l}, l \geq 2$, implies $\left|E\left(\Gamma_{n}^{k, l}\right)\right|=m+k l$. After successive applications of transformation $A$ as $A_{\alpha}$, $\alpha \leq l-1$, the edge set of $A_{\alpha}\left(\Gamma_{n}^{k, l}\right)$ is partitioned as $E_{\left(\operatorname{deg}_{s}+\operatorname{deg}_{t}\right)}$ $\left(A_{\alpha}\left(\Gamma_{n}^{k, l}\right)\right),\left(\operatorname{deg}_{s}+\operatorname{deg}_{t}\right) \in\left\{3,4, \operatorname{deg}_{u}+\alpha+2, \operatorname{deg}_{u}+\alpha+3\right.$, $\left.\operatorname{deg}_{u}+\operatorname{deg}_{v}, \operatorname{deg}_{u}+\alpha+1+\operatorname{deg}_{v}\right\}$ :

$$
\begin{aligned}
& E_{3}\left(A_{\alpha}\left(\Gamma_{n}^{k, l}\right)\right)=\left\{s t \in \Gamma_{n}^{k, l}: \operatorname{deg}_{s}=1, \operatorname{deg}_{t}=2\right\} \\
& E_{4}\left(A_{\alpha}\left(\Gamma_{n}^{k, l}\right)\right)=\left\{s t \in \Gamma_{n}^{k, l}: \operatorname{deg}_{s}=\operatorname{deg}_{t}=2\right\}, \\
& E_{\operatorname{deg}_{u}+\alpha+2}\left(A_{\alpha}\left(\Gamma_{n}^{k, l}\right)\right)=\left\{s t \in \Gamma_{n}^{k, l}: \delta_{\Gamma} \leq \operatorname{deg}_{s}=\operatorname{deg}_{u}+\alpha+1 \leq \Delta_{\Gamma}+\alpha+1, \operatorname{deg}_{t}=1\right\}, \\
& E_{\operatorname{deg}_{u}+\alpha+3}\left(A_{\alpha}\left(\Gamma_{n}^{k, l}\right)\right)=\left\{s t \in \Gamma_{n}^{k, l}: \delta_{\Gamma} \leq \operatorname{deg}_{s}=\operatorname{deg}_{u}+\alpha+1 \leq \Delta_{\Gamma}+\alpha+1, \operatorname{deg}_{t}=2\right\}, \\
& E_{\operatorname{deg}_{u}+\alpha+1}\left(A_{\alpha}\left(\Gamma_{n}^{k, l}\right)\right)=\left\{s t \in \Gamma_{n}^{k, l}: \delta_{\Gamma} \leq \operatorname{deg}_{s}=\operatorname{deg}_{u}+\alpha \leq \Delta_{\Gamma}, \operatorname{deg}_{t}=1\right\}, \\
& E_{\operatorname{deg}_{u}+\operatorname{deg}_{v}}\left(A_{\alpha}\left(\Gamma_{n}^{k, l}\right)\right)=\left\{s t \in \Gamma_{n}^{k, l}: \delta_{\Gamma} \leq \operatorname{deg}_{s}=\operatorname{deg}_{u}, \operatorname{deg}_{t}=\operatorname{deg}_{v} \leq \Delta_{\Gamma}\right\}, \\
& E_{\operatorname{deg}_{u}+\alpha+1+\operatorname{deg}_{v}}\left(A_{\alpha}\left(\Gamma_{n}^{k, l}\right)\right)=\left\{s t \in \Gamma_{n}^{k, l}: \delta_{\Gamma} \leq \operatorname{deg}_{v}, \operatorname{deg}_{u} \leq \Delta_{\Gamma}, \operatorname{deg}_{s}=\operatorname{deg}_{u}+\alpha+1, \operatorname{deg}_{t}=\operatorname{deg}_{v}\right\}, \\
& \operatorname{GA}\left(A_{\alpha}\left(\Gamma_{n}^{k, l}\right)\right)=\sum_{\substack{E_{\left(\operatorname{deg}_{s}+\operatorname{deg}_{t}\right)}\left(A_{\alpha}\left(\Gamma_{n}^{k, l}\right)\right) \\
\subseteq E\left(A_{\alpha}\left(\Gamma_{n}^{k, l}\right)\right)}} \sum \sum \frac{2 \sqrt{\operatorname{deg}_{s} \operatorname{deg}_{t}}}{{\substack{\text { st } \\
E_{\left(\operatorname{deg}_{s}+\operatorname{deg}_{t}\right)}\left(A_{\alpha}\left(\Gamma_{n}^{k, l}\right)\right)}}_{\operatorname{deg}_{s}+\operatorname{deg}_{t}}} \sum
\end{aligned}
$$


The cardinality of $A_{3}$ is $k$, i.e., $\left|E_{3}\left(A_{\alpha}\left(\Gamma_{n}^{k, l}\right)\right)\right|=k, \| E_{4}\left(A_{\alpha}\right.$ $\left.\left(\Gamma_{n}^{k, l}\right)\right)|=k(l-\alpha-2), \quad| E_{\operatorname{deg}_{u}+\alpha+2}\left(A_{\alpha}\left(\Gamma_{n}^{k, l}\right)\right) \mid=k \alpha, \quad$ and $\left|E_{\operatorname{deg}_{u}+\alpha+3}\left(A_{\alpha}\left(\Gamma_{n}^{k, l}\right)\right)\right|=k$. The function $f(x)=2 \sqrt{a x} / a+x$ is decreasing, where $a \leq x$ is a constant. So, for $\delta_{\Gamma}$ minimum degree of $\Gamma$ and $\Delta_{\Gamma}$ maximum, for any graph,

$$
\begin{aligned}
& \frac{2 \sqrt{2 *\left(\operatorname{deg}_{s}+1+\alpha\right)}}{2+\left(\operatorname{deg}_{s}+1+\alpha\right)} \geq \frac{2 \sqrt{2 *\left(\Delta_{\Gamma}+1+\alpha\right)}}{2+\left(\Delta_{\Gamma}+1+\alpha\right)}, \\
& \frac{2 \sqrt{\left(\Delta_{\Gamma}+\alpha+1\right) \delta_{\Gamma}}}{\left(\Delta_{\Gamma}+\alpha+1\right)+\delta_{\Gamma}} \leq \frac{2 \sqrt{\left(\operatorname{deg}_{s}+\alpha+1\right) \operatorname{deg}_{t}}}{\left(\operatorname{deg}_{s}+\alpha+1\right)+\operatorname{deg}_{t}}
\end{aligned}
$$

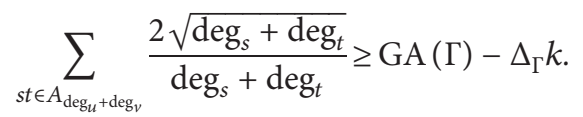

Substituting these changes in equation (24), we have the following inequality:

$$
\begin{aligned}
\mathrm{GA}\left(A_{\alpha}\left(\Gamma_{n}^{k, l}\right)\right) \geq & \frac{2 k \sqrt{2}}{3}+k(l-2-\alpha) \\
& +\frac{2 \alpha k \sqrt{1 *\left(\Delta_{\Gamma}+\alpha+1\right)}}{1+\left(\Delta_{\Gamma}+\alpha+1\right)}+\frac{2 k \sqrt{2\left(\Delta_{\Gamma}+\alpha+1\right)}}{2+\left(\Delta_{\Gamma}+\alpha+1\right)} \\
& +\frac{2 k \Delta_{\Gamma} \sqrt{\left(\Delta_{\Gamma}+\alpha+1\right) \delta_{\Gamma}}}{\left(\Delta_{\Gamma}+\alpha+1\right)+\delta_{\Gamma}}+\mathrm{GA}(\Gamma)-\Delta_{\Gamma} K .
\end{aligned}
$$

After simplification, we get the required result

$$
\begin{aligned}
\mathrm{GA}\left(A_{\alpha}\left(\Gamma_{n}^{k, l}\right)\right) \geq & 2 k \sqrt{\Delta_{\Gamma}+\alpha+1}\left[\frac{\alpha}{\Delta_{\Gamma}+\alpha+2}+\frac{\sqrt{2}}{\Delta_{\Gamma}+\alpha+3}+\frac{\Delta_{\Gamma} \sqrt{\delta_{\Gamma}}}{\left(\Delta_{\Gamma}+1+\alpha\right)+\delta_{\Gamma}}\right] \\
& +\frac{2 k \sqrt{2}}{3}+\mathrm{GA}(\Gamma)+k l-k\left(2+\alpha+\Delta_{\Gamma}\right) .
\end{aligned}
$$

Now, again, from equation (24) and inequalities,

$$
\begin{aligned}
\sum_{s t \in A_{\operatorname{deg}_{u}+\operatorname{deg}_{v}} \frac{2 \sqrt{\operatorname{deg}_{s}+\operatorname{deg}_{t}}}{\operatorname{deg}_{s}+\operatorname{deg}_{t}} \geq \operatorname{GA}(\Gamma)-\frac{2 \Delta_{\Gamma} \sqrt{\Delta_{\Gamma} \delta_{\Gamma}}}{\Delta_{\Gamma}+\delta_{\Gamma}},} \\
\frac{2 \sqrt{\operatorname{deg}_{s}+\alpha+1}}{1+\left(\operatorname{deg}_{s}+\alpha+1\right)} \leq \frac{2 \sqrt{\delta_{\Gamma}+\alpha+1}}{1+\left(\delta_{\Gamma}+\alpha+1\right)}, \\
\frac{2 \Delta_{\Gamma} k \sqrt{\left(\Delta_{\Gamma}+1\right) \Delta_{\Gamma}}}{\left(\Delta_{\Gamma}+1\right)+\Delta_{\Gamma}} \geq \sum_{s t \in A_{\operatorname{deg}_{u}+\operatorname{deg}_{v}+\alpha+1}} \frac{2 \sqrt{\left(\operatorname{deg}_{s}+\alpha+1\right) \operatorname{deg}_{t}}}{\left(\operatorname{deg}_{s}+\alpha+1\right)+\operatorname{deg}_{t}}, \\
\frac{2 \sqrt{2 *\left(\operatorname{deg}_{s}+\alpha+1\right)}}{2+\left(\operatorname{deg}_{s}+\alpha+1\right)} \leq \frac{2 \sqrt{2 *\left(\delta_{\Gamma}+\alpha+1\right)}}{2+\left(\delta_{\Gamma}+\alpha+1\right)},
\end{aligned}
$$

$$
\begin{aligned}
\mathrm{GA}\left(A_{\alpha}\left(\Gamma_{n}^{k, l}\right)\right) \leq & \frac{2 k \sqrt{2}}{3}+\frac{2 \alpha k \sqrt{1 *\left(\delta_{\Gamma}+\alpha+1\right)}}{1+\left(\delta_{\Gamma}+\alpha+1\right)} \\
& +\frac{2 k \sqrt{2 *\left(\delta_{\Gamma}+\alpha+1\right)}}{2+\left(\delta_{\Gamma}+\alpha+1\right)}+\frac{2 \Delta_{\Gamma} k \sqrt{\left(\Delta_{\Gamma}+1\right) \Delta_{\Gamma}}}{\left(\Delta_{\Gamma}+1\right)+\Delta_{\Gamma}} \\
& +k(l-2-\alpha)+\mathrm{GA}(\Gamma)-\frac{2 k \Delta_{\Gamma} \sqrt{\Delta_{\Gamma} \delta_{\Gamma}}}{\Delta_{\Gamma}+\delta_{\Gamma}} .
\end{aligned}
$$

After simplification, we obtain

$$
\begin{aligned}
\mathrm{GA}\left(A_{\alpha}\left(\Gamma_{n}^{k, l}\right)\right) \leq & 2 k \sqrt{\delta_{\Gamma}+\alpha+1}\left[\frac{\alpha}{\delta_{\Gamma}+\alpha+2}+\frac{\sqrt{2}}{\delta_{\Gamma}+\alpha+3}\right] \\
& +\frac{2 k \sqrt{2}}{3}+k(l-2-\alpha)+\mathrm{GA}(\Gamma)+2 k \Delta_{\Gamma}^{3 / 3} \\
& \cdot\left[\frac{\sqrt{\Delta_{\Gamma}+1}}{2 \Delta_{\Gamma}+1}-\frac{\sqrt{\delta_{\Gamma}}}{\Delta_{\Gamma}+\delta_{\Gamma}}\right] .
\end{aligned}
$$

Inequalities (27) and (30) complete the proof.

Transformation $B$ : let $w_{j} \in V(\Gamma), \operatorname{deg}_{w_{j}} \geq 2$, for $1 \leq j \leq k \leq n$, and paths pendent at $w_{j}$ of the form $\left\{w_{j} u_{j}^{1}, u_{j}^{1} u_{j}^{2}, u_{j}^{2} u_{j}^{3}, \ldots, u_{j}^{l-1} u_{j}^{l}\right\}$ which comprises $\Gamma_{n}^{k, l}$. Then, for fixed vertex $w_{1}$,

$$
\begin{aligned}
B\left(\Gamma_{n}^{k, l}\right)= & \Gamma_{n}^{k, l}-\left\{u_{j}^{1} u_{j}^{2}, u_{j}^{2} u_{j}^{3}, \ldots, u_{j}^{l-1} u_{j}^{l}\right\} \\
& +\left\{w_{1} u_{j}^{1}, u_{j}^{1} u_{j}^{2}, u_{j}^{2} u_{j}^{3}, \ldots, u_{j}^{l-1} u_{j}^{l}\right\} .
\end{aligned}
$$

The transformation $B$ is shown in Figure 3 and $A_{\alpha}^{\beta}$ shown in Figure 4.

Transformation $A_{\alpha}^{\beta}$ : let $0 \leq \alpha \leq l-1$ and $0 \leq \beta \leq k-1$. The transformation $A_{\alpha}^{\beta}$ is the composition of successive applications of transformation $A$ and $B$ as $A_{\alpha}$ and $B_{\beta}$, respectively [27].

In Theorem 3, we discuss the effect of transformation $A_{\alpha}^{\beta}$ over the $G A$ index.

Theorem 3. Let graph $\Gamma_{n}^{k, l}$ comprise of n-vertex simple connected graph $\Gamma$ along with $k$ pendent paths of length $l \geq 2$ attached with $v \in \Gamma$ of degree $d_{v} \geq 2$, maximum degree of $v \in \Gamma_{n}^{k, l}$ is $\Delta_{\Gamma}+1$, and minimum $\delta_{\Gamma}$. Then, 


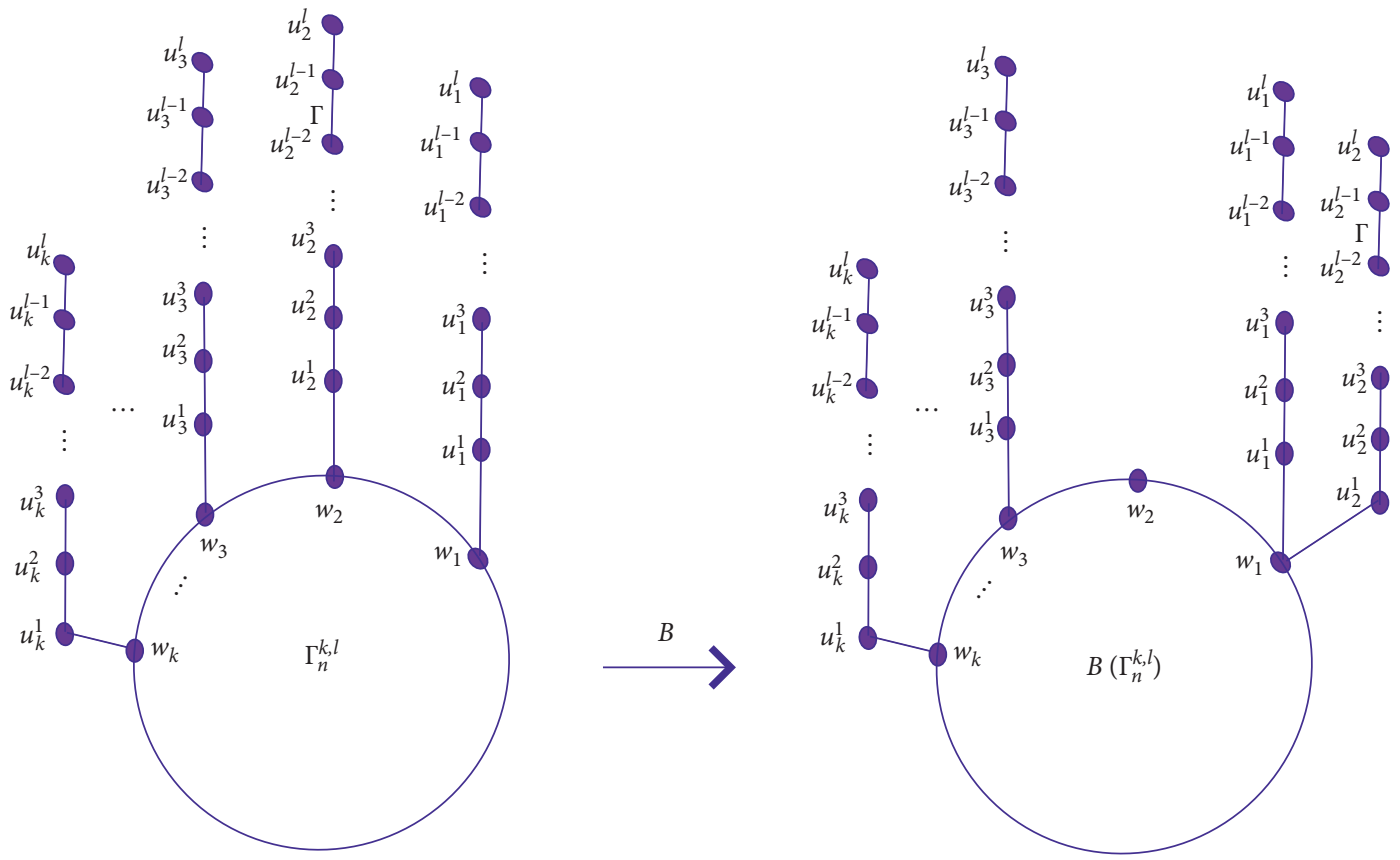

Figure 3: Transformation $B$ for fixed vertex $w_{1}$.

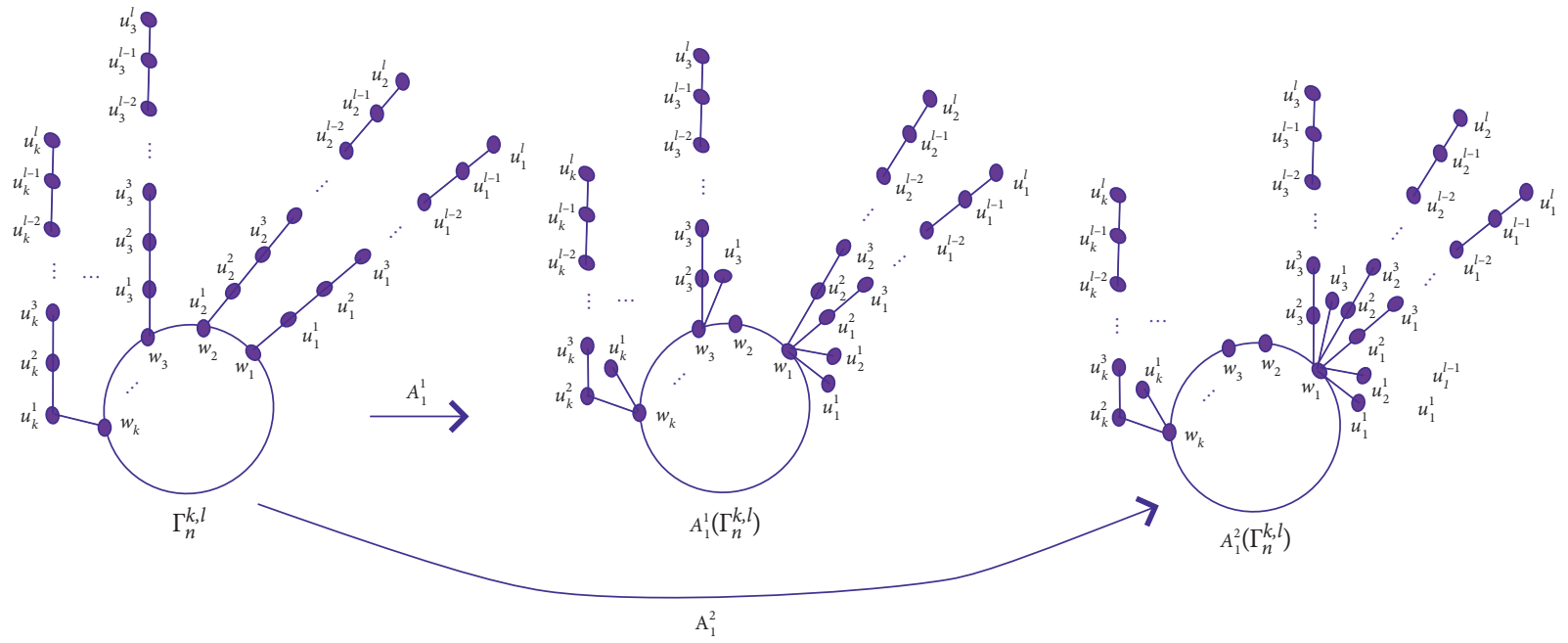

Figure 4: Transformation $A_{\alpha}^{\beta}$.

$$
\begin{aligned}
\operatorname{GA}\left(A_{\alpha}^{\beta}\left(\Gamma_{n}^{k, l}\right)\right) \geq 2 & (k-\beta-1) \sqrt{\Delta_{\Gamma}+\alpha+1}\left(\frac{\alpha}{\Delta_{\Gamma}+\alpha+2}+\frac{\sqrt{2}}{\Delta_{\Gamma}+\alpha+3}+\frac{\Delta_{\Gamma} \sqrt{\delta_{\Gamma}}}{\Delta_{\Gamma}+\alpha+1+\delta_{\Gamma}}\right) \\
& +2 \sqrt{\Delta_{\Gamma}+(\beta+1)(1+\alpha)}\left(\frac{\alpha(\beta+1)}{1+\Delta_{\Gamma}+(\beta+1)(1+\alpha)}+\frac{\sqrt{2}(\beta+1)}{2+\Delta_{\Gamma}+(\beta+1)(1+\alpha)}\right) \\
& +2 \frac{\Delta_{\Gamma} \sqrt{\left(\Delta_{\Gamma}+(\beta+1)(1+\alpha)\right) \delta_{\Gamma}}}{\delta_{\Gamma}+\Delta_{\Gamma}+(\beta+1)(1+\alpha)}+k(l-2-\alpha)-\Delta_{\Gamma}(k-\beta)+\frac{2 k \sqrt{2}}{3}+\mathrm{GA}(\Gamma),
\end{aligned}
$$




$$
\begin{aligned}
\mathrm{GA}\left(A_{\alpha}^{\beta}\left(\Gamma_{n}^{k, l}\right)\right) \leq & 2(k-\beta-1) \sqrt{\delta_{\Gamma}+\alpha+1}\left(\frac{\alpha}{\delta_{\Gamma}+\alpha+2}+\frac{\sqrt{2}}{\delta_{\Gamma}+\alpha+3}\right)+k(l-2-\alpha)+\frac{2 k \sqrt{2}}{3} \\
& +2 \sqrt{\delta_{\Gamma}+(\beta+1)(1+\alpha)}\left(\frac{\alpha(\beta+1)}{1+\delta_{\Gamma}+(\beta+1)(1+\alpha)}+\frac{\sqrt{2}(\beta+1)}{2+\delta_{\Gamma}+(\beta+1)(1+\alpha)}\right) \\
& +2 \Delta_{\Gamma} \sqrt{\Delta_{\Gamma}}(k-\beta)\left(\frac{\sqrt{\Delta_{\Gamma}+1}}{2 \Delta_{\Gamma}+1}-\frac{\sqrt{\delta_{\Gamma}}}{\Delta_{\Gamma}+\delta_{\Gamma}}\right)+\mathrm{GA}(\Gamma) .
\end{aligned}
$$
$\beta=0$.

Equality holds for graph of the type II with $\alpha=0$ and

Proof. Let a simple graph $\Gamma$ of order $n$, size $m$, minimum degree $\delta_{\Gamma}$, and maximum $\Delta_{\Gamma}$. Let $\Gamma_{n}^{k, l}$ be the graph formed by $k$ number of paths of length $l$ pendent at distinct fully connected vertices of $\Gamma$. The geometric-arithmetic index of any graph $\Gamma$ is

$$
\mathrm{GA}(\Gamma)=\sum_{s t \in E(G)} \frac{2 \sqrt{\operatorname{deg}_{s} \operatorname{deg}_{t}}}{\operatorname{deg}_{s}+\operatorname{deg}_{t}}
$$

The construction of $\Gamma_{n}^{k, l}, l \geq 2$, implies $\left|E\left(\Gamma_{n}^{k, l}\right)\right|=m+k l$. Let $u^{*}$ be the fixed vertex. Applications of transformation $A_{\alpha}^{\beta}$ has an effect over the edge set partition as $E_{\left(\operatorname{deg}_{s}+\operatorname{deg}_{t}\right)}\left(A_{\alpha}^{\beta}\right.$ $\left.\left(\Gamma_{n}^{k, l}\right)\right), \quad\left(\operatorname{deg}_{s}+\operatorname{deg}_{t}\right) \in\left\{3,4, \operatorname{deg}_{u}+\alpha+2, \operatorname{deg}_{u}+\alpha+3, \operatorname{deg}_{u}+\right.$ $\operatorname{deg}_{v}, \operatorname{deg}_{u}+\alpha+1+\operatorname{deg}_{v}, \operatorname{deg}_{u^{*}}+(\beta+1)(\alpha+1)+\operatorname{deg}_{v}, \operatorname{deg}_{u^{*}}+$ $\left.(\beta+1)(\alpha+1)+1, \operatorname{deg}_{u^{*}}+(\beta+1)(\alpha+1)+2\right\}$ :

$$
\begin{aligned}
& E_{3}\left(A_{\alpha}^{\beta}\left(\Gamma_{n}^{k, l}\right)\right)=\left\{s t \in \Gamma_{n}^{k, l}: \operatorname{deg}_{s}=1, \operatorname{deg}_{t}=2\right\} \\
& E_{4}\left(A_{\alpha}^{\beta}\left(\Gamma_{n}^{k, l}\right)\right)=\left\{s t \in \Gamma_{n}^{k, l}: \operatorname{deg}_{s}=\operatorname{deg}_{t}=2\right\}, \\
& E_{\operatorname{deg}_{u}+\alpha+2}\left(A_{\alpha}^{\beta}\left(\Gamma_{n}^{k, l}\right)\right)=\left\{s t \in \Gamma_{n}^{k, l}: \delta_{\Gamma} \leq \operatorname{deg}_{s}=\operatorname{deg}_{u}+\alpha+1 \leq \Delta_{\Gamma}+\alpha+1, \operatorname{deg}_{t}=1, E_{\operatorname{deg}_{u}+\alpha+3} A_{\alpha}^{\beta}\left(\Gamma_{n}^{k, l}\right)\right\} \\
& =\left\{s t \in \Gamma_{n}^{k, l}: \delta_{\Gamma} \leq \operatorname{deg}_{s}=\operatorname{deg}_{u}+\alpha+1 \leq \Delta_{\Gamma}+\alpha+1, \operatorname{deg}_{t}=2, E_{\operatorname{deg}_{u}+\operatorname{deg}_{v}} A_{\alpha}^{\beta}\left(\Gamma_{n}^{k, l}\right)\right\} \\
& =\left\{s t \in \Gamma_{n}^{k, l}: \delta_{\Gamma} \leq \operatorname{deg}_{s}=\operatorname{deg}_{u}, \operatorname{deg}_{t}=\operatorname{deg}_{v} \leq \Delta_{\Gamma}\right\}, \\
& E_{\operatorname{deg}_{u}+\alpha+1+\operatorname{deg}_{v}}\left(A_{\alpha}^{\beta}\left(\Gamma_{n}^{k, l}\right)\right)=\left\{s t \in \Gamma_{n}^{k, l}: \delta_{\Gamma} \leq \operatorname{deg}_{v}=\operatorname{deg}_{t}, \operatorname{deg}_{u} \leq \Delta_{\Gamma}, \operatorname{deg}_{s}=\operatorname{deg}_{u}+\alpha+1\right\}, \\
& E_{\operatorname{deg}_{u^{*}}+(\beta+1)(\alpha+1)+\operatorname{deg}_{v}}\left(A_{\alpha}^{\beta}\left(\Gamma_{n}^{k, l}\right)\right)=\left\{s t \in \Gamma_{n}^{k, l}: \delta_{\Gamma} \leq \operatorname{deg}_{v}=\operatorname{deg}_{t}, \operatorname{deg}_{u} \leq \Delta_{\Gamma}, \operatorname{deg}_{s}=\operatorname{deg}_{u}+(\beta+1)(\alpha+1)\right\}, \\
& E_{\operatorname{deg}_{u^{*}}+(\beta+1)(\alpha+1)+1}\left(A_{\alpha}^{\beta}\left(\Gamma_{n}^{k, l}\right)\right)=\left\{s t \in \Gamma_{n}^{k, l}: \delta_{\Gamma} \leq \operatorname{deg}_{v}, \operatorname{deg}_{u} \leq \Delta_{\Gamma}, \operatorname{deg}_{s}=\operatorname{deg}_{u}+(\beta+1)(\alpha+1), \operatorname{deg}_{t}=1\right\}, \\
& E_{\operatorname{deg}_{u^{*}}(\beta+1)(\alpha+1)+2}\left(A_{\alpha}^{\beta}\left(\Gamma_{n}^{k, l}\right)\right)=\left\{s t \in \Gamma_{n}^{k, l}: \delta_{\Gamma} \leq \operatorname{deg}_{v}, \operatorname{deg}_{u} \leq \Delta_{\Gamma}, \operatorname{deg}_{s}=\operatorname{deg}_{u}+(\beta+1)(\alpha+1), \operatorname{deg}_{t}=2\right\},
\end{aligned}
$$

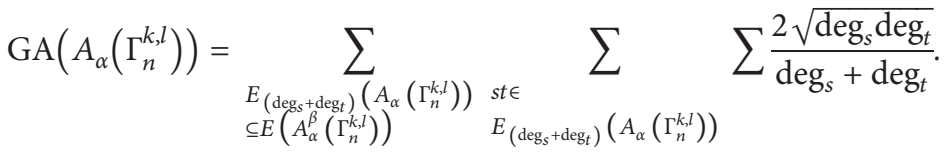

The cardinality of $E_{3}$ is $k$, i.e., $\left|E_{3}\left(A_{\alpha}^{\beta}\left(\Gamma_{n}^{k, l}\right)\right)\right|=k, \mid E_{4}$ $\left(A_{\alpha}^{\beta}\left(\Gamma_{n}^{k, l}\right)\right)|=k(l-\alpha-2),| E_{\operatorname{deg}_{u}+\alpha+2}\left(A_{\alpha}^{\beta}\left(\Gamma_{n}^{k, l}\right)\right) \mid=\alpha(k-\beta-$ 1), $\left|E_{\operatorname{deg}_{u}+\alpha+3}\left(A_{\alpha}^{\beta}\left(\Gamma_{n}^{k, l}\right)\right)\right|=k-\beta-1, \quad \mid E_{\operatorname{deg}_{u^{*}}+(\beta+1)(\alpha+1)+1}$ $\left(A_{\alpha}^{\beta}\left(\Gamma_{n}^{k, l}\right)\right) \mid=\alpha(\beta+1)$, and

$$
\left|E_{\operatorname{deg}_{u^{*}}(\beta+1)(\alpha+1)+2}\left(A_{\alpha}^{\beta}\left(\Gamma_{n}^{k, l}\right)\right)\right|=\beta+1 .
$$

The function $f(x)=2 \sqrt{a x} / a+x$ is decreasing, where $a \leq x$ is a constant. So, for $\delta_{\Gamma}$ minimum degree of $\Gamma$ and $\Delta_{\Gamma}$ maximum, we have 


$$
\begin{aligned}
& \frac{2 \sqrt{2\left(\operatorname{deg}_{s}+1+\alpha\right)}}{2+\left(\operatorname{deg}_{s}+1+\alpha\right)} \geq \frac{2 \sqrt{2\left(\Delta_{\Gamma}+1+\alpha\right)}}{2+\left(\Delta_{\Gamma}+1+\alpha\right)}, \frac{2 \sqrt{\left(\Delta_{\Gamma}+\alpha+1\right) * 1}}{\left(\Delta_{\Gamma}+\alpha+1\right)+1} \leq \frac{2 \sqrt{\left(\operatorname{deg}_{s}+\alpha+1\right) \operatorname{deg}_{t}}}{\left(\operatorname{deg}_{s}+\alpha+1\right)+\operatorname{deg}_{t}} \\
& \frac{2 \sqrt{2 *\left(\operatorname{deg}_{s}+(\beta+1)(1+\alpha)\right)}}{2+\left(\operatorname{deg}_{s}+(\beta+1)(1+\alpha)\right)} \geq \frac{2 \sqrt{2 *\left(\Delta_{\Gamma}+(\beta+1)(1+\alpha)\right)}}{2+\left(\Delta_{\Gamma}+(\beta+1)(1+\alpha)\right)}, \\
& 2 \sqrt{\left(\Delta_{\Gamma}+(\beta+1)(1+\alpha)\right) \delta_{\Gamma}} \quad 2 \sqrt{\left(\operatorname{deg}_{s}+(\beta+1)(1+\alpha)\right) \operatorname{deg}_{t}} \\
& \overline{\left(\Delta_{\Gamma}+(\beta+1)(1+\alpha)\right)+\delta_{\Gamma}} \leq \frac{\gamma}{\left(\operatorname{deg}_{s}+(\beta+1)(1+\alpha)\right)+\operatorname{deg}_{t}},
\end{aligned}
$$

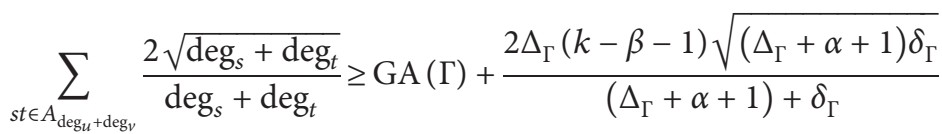

$$
\begin{aligned}
& +\frac{2 \Delta_{\Gamma} \sqrt{\left(\Delta_{\Gamma}+(\beta+1)(1+\alpha)\right) \delta_{\Gamma}}}{\left(\Delta_{\Gamma}+(\beta+1)(1+\alpha)\right)+\delta_{\Gamma}}-\Delta_{\Gamma}(k-\beta) .
\end{aligned}
$$

Substituting these changes in equation (35), we obtained the following inequality:

$$
\begin{aligned}
\mathrm{GA}\left(A_{\alpha}^{\beta}\left(\Gamma_{n}^{k, l}\right)\right) \geq & \frac{2 k \sqrt{2}}{3}+\frac{2 \alpha(k-\beta-1) \sqrt{1 *\left(\Delta_{\Gamma}+\alpha+1\right)}}{1+\left(\Delta_{\Gamma}+\alpha+1\right)}+\frac{2(k-\beta-1) \sqrt{2\left(\Delta_{\Gamma}+\alpha+1\right)}}{2+\left(\Delta_{\Gamma}+\alpha+1\right)} \\
& +\frac{2 \alpha(\beta+1) \sqrt{1 *\left(\Delta_{\Gamma}+(\beta+1)(1+\alpha)\right)}}{1+\left(\Delta_{\Gamma}+(\beta+1)(1+\alpha)\right)} \\
& +\frac{2(\beta+1) \sqrt{2\left(\Delta_{\Gamma}+(\beta+1)(1+\alpha)\right)}}{2+\left(\Delta_{\Gamma}+(\beta+1)(1+\alpha)\right)} \\
& +\frac{2 \Delta_{\Gamma}(k-\beta-1) \sqrt{\left(\Delta_{\Gamma}+\alpha+1\right) \delta_{\Gamma}}}{\left(\Delta_{\Gamma}+\alpha+1\right)+\delta_{\Gamma}}+\frac{2 \Delta_{\Gamma} \sqrt{\left(\Delta_{\Gamma}+(\beta+1)(1+\alpha)\right) \delta_{\Gamma}}}{\left(\Delta_{\Gamma}+(\beta+1)(1+\alpha)\right)+\delta_{\Gamma}} \\
& +\mathrm{GA}(\Gamma)+k(l-2-\alpha)-\Delta_{\Gamma}(k-\beta) .
\end{aligned}
$$


After simplification, we get the required result:

$$
\begin{aligned}
\mathrm{GA}\left(A_{\alpha}^{\beta}\left(\Gamma_{n}^{k, l}\right)\right) \geq 2 & (k-\beta-1) \sqrt{\delta_{\Gamma}+\alpha+1}\left(\frac{\alpha}{\Delta_{\Gamma}+\alpha+2}+\frac{\sqrt{2}}{\Delta_{\Gamma}+\alpha+3}+\frac{\Delta_{\Gamma} \sqrt{\delta_{\Gamma}}}{\Delta_{\Gamma}+\alpha+1+\delta_{\Gamma}}\right)+2 \sqrt{\Delta_{\Gamma}+(\beta+1)(1+\alpha)} \\
& \times\left(\frac{\alpha(\beta+1)}{1+\Delta_{\Gamma}+(\beta+1)(1+\alpha)}+\frac{\sqrt{2}(\beta+1)}{2+\Delta_{\Gamma}+(\beta+1)(1+\alpha)}\right)+2 \frac{\Delta_{\Gamma} \sqrt{\left(\Delta_{\Gamma}+(\beta+1)(1+\alpha)\right) \delta_{\Gamma}}}{\delta_{\Gamma}+\Delta_{\Gamma}+(\beta+1)(1+\alpha)} \\
& +k(l-2-\alpha)-\Delta_{\Gamma}(k-\beta)+\frac{2 k \sqrt{2}}{3}+\operatorname{GA}(\Gamma) .
\end{aligned}
$$

Now, again substituting the following inequalities in equation (35),

$$
\begin{aligned}
& 2 \sqrt{2\left(\operatorname{deg}_{s}+1+\alpha\right)} 2 \sqrt{2\left(\delta_{\Gamma}+1+\alpha\right)}
\end{aligned}
$$

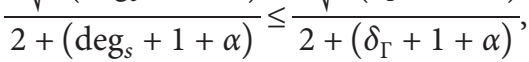

$$
\begin{aligned}
& \frac{2 \sqrt{\left(\operatorname{deg}_{s}+\alpha+1\right) \operatorname{deg}_{t}}}{\left(\operatorname{deg}_{s}+\alpha+1\right)+\operatorname{deg}_{t}} \leq \frac{2 \sqrt{\left(\delta_{\Gamma}+\alpha+1\right)}}{\left(\delta_{\Gamma}+\alpha+1\right)+1}
\end{aligned}
$$

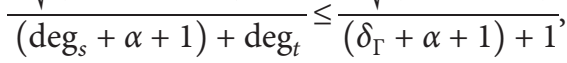

$$
\begin{aligned}
& 2 \sqrt{2\left(\operatorname{deg}_{s}+(\beta+1)(1+\alpha)\right)} \leq 2 \sqrt{2\left(\delta_{\Gamma}+(\beta+1)(1+\alpha)\right)} \\
& \overline{2+\left(\operatorname{deg}_{s}+(\beta+1)(1+\alpha)\right)} \leq \frac{1}{2+\left(\delta_{\Gamma}+(\beta+1)(1+\alpha)\right)}, \\
& \frac{2 \sqrt{\left(\operatorname{deg}_{s}+(\beta+1)(1+\alpha)\right)}}{1+\left(\operatorname{deg}_{s}+(\beta+1)(1+\alpha)\right)} \leq \frac{2 \sqrt{\left(\delta_{\Gamma}+(\beta+1)(1+\alpha)\right)}}{1+\left(\delta_{\Gamma}+(\beta+1)(1+\alpha)\right)}
\end{aligned}
$$

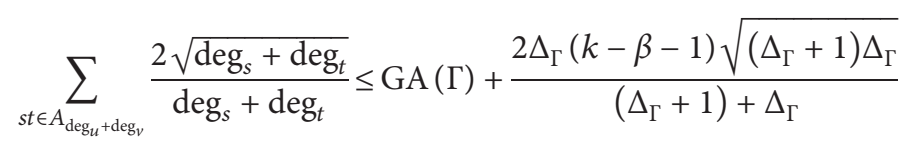

$$
\begin{aligned}
& +\frac{2 \Delta_{\Gamma} \sqrt{\left(\Delta_{\Gamma}+1\right) \Delta_{\Gamma}}}{\left(\Delta_{\Gamma}+1\right)+\Delta_{\Gamma}}-\frac{2 \Delta_{\Gamma}(k-\beta) \sqrt{\Delta_{\Gamma} \delta_{\Gamma}}}{\Delta_{\Gamma}+\delta_{\Gamma}} .
\end{aligned}
$$


We obtain

$$
\begin{aligned}
\mathrm{GA}\left(A_{\alpha}^{\beta}\left(\Gamma_{n}^{k, l}\right)\right) \leq & \frac{2 k \sqrt{2}}{3}+\frac{2 \alpha(k-\beta-1) \sqrt{1 *\left(\delta_{\Gamma}+\alpha+1\right)}}{1+\left(\delta_{\Gamma}+\alpha+1\right)}+\frac{2(k-\beta-1) \sqrt{2\left(\delta_{\Gamma}+\alpha+1\right)}}{2+\left(\delta_{\Gamma}+\alpha+1\right)} \\
& +\frac{2 \alpha(\beta+1) \sqrt{1 *\left(\delta_{\Gamma}+(\beta+1)(1+\alpha)\right)}}{1+\left(\delta_{\Gamma}+(\beta+1)(1+\alpha)\right)} \\
& +\frac{2(\beta+1) \sqrt{2\left(\delta_{\Gamma}+(\beta+1)(1+\alpha)\right)}}{2+\left(\delta_{\Gamma}+(\beta+1)(1+\alpha)\right)}+\mathrm{GA}(\Gamma)+\frac{2 \Delta_{\Gamma}(k-\beta-1) \sqrt{\left(\Delta_{\Gamma}+1\right) \Delta_{\Gamma}}}{\left(\Delta_{\Gamma}+1\right)+\Delta_{\Gamma}} \\
& +\frac{2 \Delta_{\Gamma} \sqrt{\left(\Delta_{\Gamma}+1\right) \Delta_{\Gamma}}}{\left(\Delta_{\Gamma}+1\right)+\Delta_{\Gamma}}-\frac{2 \Delta_{\Gamma}(k-\beta) \sqrt{\Delta_{\Gamma} \delta_{\Gamma}}}{\Delta_{\Gamma}+\delta_{\Gamma}} .
\end{aligned}
$$

After simplification, we get the required result:

$$
\begin{aligned}
\mathrm{GA}\left(A_{\alpha}^{\beta}\left(\Gamma_{n}^{k, l}\right)\right) \leq & 2(k-\beta-1) \sqrt{\delta_{\Gamma}+\alpha+1}\left(\frac{\alpha}{\delta_{\Gamma}+\alpha+2}+\frac{\sqrt{2}}{\delta_{\Gamma}+\alpha+3}\right)+k(l-2-\alpha)+\frac{2 k \sqrt{2}}{3} \\
& +2 \sqrt{\delta_{\Gamma}+(\beta+1)(1+\alpha)}\left(\frac{\alpha(\beta+1)}{1+\delta_{\Gamma}+(\beta+1)(1+\alpha)}+\frac{\sqrt{2}(\beta+1)}{2+\delta_{\Gamma}+(\beta+1)(1+\alpha)}\right) \\
& +2 \Delta_{\Gamma} \sqrt{\Delta_{\Gamma}}(k-\beta)\left(\frac{\sqrt{\Delta_{\Gamma}+1}}{2 \Delta_{\Gamma}+1}-\frac{\sqrt{\delta_{\Gamma}}}{\Delta_{\Gamma}+\delta_{\Gamma}}\right)+\mathrm{GA}(\Gamma) .
\end{aligned}
$$

Inequalities (40) and (43) complete the proof.

\section{Conclusion}

The study of mathematical aspect regarding topological indices is a partially open problem $[20,28,29]$. For which member family of graphs the certain index has a minimal or maximal value? In this work, we discussed, for this fundamental question, general graphs with pendent paths for the most studied index named geometric-arithmetic index GA and developed tight bounds by characterizing graphs. In Theorems 2 and 3, for the first time, we defined tight bonds for the transformed graphs under the effect of transformations defined in [27].

\section{Data Availability}

No data were used to support this study.

\section{Disclosure}

This paper has not been published elsewhere and it will not be submitted anywhere else for publication.

\section{Conflicts of Interest}

The authors declare that they have no conflicts of interest.

\section{Authors' Contributions}

All authors have equally contributed to the study.

\section{Acknowledgments}

The authors extend their appreciation to the deputyship for Research and Innovation, Ministry of Education in Saudi Arabia, for funding this research work through the project no. IFP-2020-17.

\section{References}

[1] L. N. Bhuyan, Q. Yang, and D. P. Agrawal, "Performance of multiprocessor interconnection networks," Computer, vol. 22, no. 2, pp. 25-37, 1989.

[2] T. y. Feng, "A survey of interconnection networks," Computer, vol. 14, no. 12, pp. 12-27, 1981.

[3] J. A. Bondy and U. S. R. Murty, Graph Theory with Applications, Macmillan, London, UK, 1976.

[4] M. Imran, S. Hayat, and M. Y. H. Mailk, "On topological indices of certain interconnection networks," Applied Mathematics and Computation, vol. 244, pp. 936-951, 2014.

[5] M. Imran, A. Q. Baig, and H. Ali, "On topological properties of dominating David derived networks," Canadian Journal of Chemistry, vol. 94, no. 2, pp. 137-148, 2016. 
[6] M. Imran, M. A. Iqbal, Y. Liu, A. Q. Baig, W. Khalid, and M. A. Zaighum, "Computing eccentricity-based topological indices of 2-power interconnection networks," Journal of Chemistry, vol. 2020, Article ID 3794592, 2020.

[7] N. Trinajstic, Chemical Graph Theory, Routledge, England, UK, 2018.

[8] J.-B. Liu, H. Shaker, I. Nadeem, and M. Hussain, "Topological aspects of Boron nanotubes," Advances in Materials Science and Engineering, vol. 2018, Article ID 5729291, 2018.

[9] M. Asif, M. Hussain, H. Almohamedh et al., "Study of carbon nanocones via connection zagreb indices," Mathematical Problems in Engineering, vol. 2021, Article ID 5539904, 2021.

[10] A. A. Dobrynin, R. Entringer, and I. Gutman, "Wiener index of trees: theory and applications," Acta Applicandae Mathematicae, vol. 66, no. 3, pp. 211-249, 2001.

[11] G. Rücker and C. Rücker, "On topological indices, boiling points, and cycloalkanes," Journal of Chemical Information and Computer Sciences, vol. 39, no. 5, pp. 788-802, 1999.

[12] S. Mondal, A. Dey, N. De, and A. Pal, "QSPR analysis of some novel neighbourhood degree-based topological descriptors," Complex and Intelligent Systems, vol. 7, no. 2, pp. 977-996, 2021.

[13] H. Ahmad, M. Hussain, W. Nazeer, and Y. M. Chu, "Distance based invariants of zigzag polyhex nanotube," Mathematical Methods in the Applied Sciences, vol. 2021, pp. 1-21.

[14] H. Wiener, "Structural determination of paraffin boiling points," Journal of the American Chemical Society, vol. 69, no. 1, pp. 17-20, 1947.

[15] M. Randic, "Characterization of molecular branching," Journal of the American Chemical Society, vol. 97, no. 23, pp. 6609-6615, 1975.

[16] D. Vukičević and B. Furtula, "Topological index based on the ratios of geometrical and arithmetical means of end-vertex degrees of edges," Journal of Mathematical Chemistry, vol. 46, pp. 1369-1376, 2009.

[17] Y. Yuan, B. Zhou, and N. Trinajstić, "On geometric-arithmetic index," Journal of Mathematical Chemistry, vol. 47, no. 2, pp. 833-841, 2010.

[18] K. C. Das and N. Trinajstić, "Comparison between first geometric-arithmetic index and atom-bond connectivity index," Chemical Physics Letters, vol. 497, no. 1-3, pp. 149-151, 2010.

[19] H. Hua and S. Zhang, "A unified approach to extremal trees with respect to geometric-arithmetic, Szeged and edge Szeged indices," MATCH Communications in Mathematical and in Computer Chemistry, vol. 65, pp. 691-704, 2011.

[20] T. Divnić, M. Milivojević, and L. Pavlović, "Extremal graphs for the geometric-arithmetic index with given minimum degree," Discrete Applied Mathematics, vol. 162, pp. 386-390, 2014.

[21] J. Rodriguez and J. Sigarreta, "On the geometric-arithmetic index," MATCH Communications in Mathematical and in Computer Chemistry, vol. 74, pp. 103-120, 2015.

[22] J. M. Sigarreta, "Bounds for the geometric-arithmetic index of a graph," Miskolc Mathematical Notes, vol. 16, no. 2, pp. 1199-1212, 2015.

[23] A. Portilla, J. Rodriguez, and J. Sigaretta, "Recent lower bounds for geometric-arithmetic index," Discrete Mathematics Letters, vol. 1, pp. 59-82, 2019.

[24] R. Hasni and N. H. M. Husin, "Bicyclic graphs with maximum geometric-arithmetic index," Applied Mathematics E-Notes, vol. 20, pp. 8-32, 2020.
[25] K. C. Das, "On geometric-arithmetic index of graphs," MATCH Communications in Mathematical and in Computer Chemistry, vol. 64, pp. 619-630, 2010.

[26] K. C. Das, I. Gutman, and B. Furtula, "Survey on geometricarithmetic indices of graphs," MATCH Communications in Mathematical and in Computer Chemistry, vol. 65, pp. 595644, 2011.

[27] M. Asif, M. Hussain, H. Almohamedh, K. M. Alhamed, and S. Almotairi, "An approach to the extremal inverse degree index for families of graphs with transformation effect," Journal of Chemistry, vol. 2021, Article ID 6657039, 2021.

[28] A. Ali and D. Dimitrov, "On the extremal graphs with respect to bond incident degree indices," Discrete Applied Mathematics, vol. 238, pp. 32-40, 2018.

[29] A. Ali, L. Zhong, and I. Gutman, "Harmonic index and its generalizations: extremal results and bounds," MATCH Communications in Mathematical and in Computer Chemistry, vol. 81, pp. 249-311, 2019. 